\title{
¿Pueden prosperar los países ricos sin que progresen los países pobres?
}

Existen cuatro problemas económicos separados pero interrelacionados, que se plantean hoy día a los formuladores de políticas cuando estudian cómo establecer una economía mundial ventajosa para todos.

El primer problema es cómo restablecer el crecimiento y el pro greso en los paises industrializados, que parecen actualmente.incapaces de recobrar el alto nivel de empleo y la baja inflación del último decenio. Es más, se prevé que las tasas de crecimiento en dichos países no superarán el 36 el 4 por ciento, mientras el desempleo y la inflación permanecerán a niveles sin precedentes en la postguerra.

En segundo lugar, cómo es posible acomodlarse a los superávit de los países exportadores de petróleo. Los excedentes de capital de la OPEP, la mayoría de los cuales se depositan a corto plazo en los países industrializados, deben utilizarse para inversiones productivas a largo plazo, con vistas a reactivar el crecimiento económico mundial.

El tercer problema consiste en cómo ayudar a los paises en desanollo de ingresos medios a recuperar el notable ritmo de crecimiento que lograron en los años 60 y principios de los 70 , a fin de que puedan guardar alguna esperanza de satisfacer las necesidades de sus poblaciones durante los próximos decenios.

El cuarto y último problema es el que plantean las sombrias perspectivas de desarrollo de los países más pobres, que se enfrentarán con una estagnación continua, a menos que se adopten medidas especiales para que se desarrollen en forma sostenida.

El cuadro que figura a continuación indica las perspectivas económicas alternativas para los países desarrollados y en desarrollo hasta el año 2000. Las cifras más elevadas se basan en proyecciones del Banco Mundial realizadas en 1976 y extendidas hasta final de siglo mediante la proyección de las tasas de crecimiento de 1975-78 estimadas por el Banco. Las tasas de crecimiento per cápita anuales son del 1,6 por ciento para los paises más pobres, 3,9 por ciento para los páses de ingresos medios y 4 por ciento para los países desa-

* Documento preparado originalmente para el Overseas Development Council, de Washington D.C. Consultado con James P. Grant y James W. Howe, y confeccionado con la colaboración de Stephen Taran, Rosemary Philips y Dorothy Seavy. 
rrollados. Las cifras más bajas proyectan una tasa de crecimiento de la nitad solamente.

\section{PERSPECTIVAS ECONONICAS GLOBALES}

Ingresos per cápita

(en dólares EE.UU. constantes del año 1975)

\begin{tabular}{lcccc}
\hline & 1965 & 1975 & 1985 & 2000 \\
\hline $\begin{array}{l}\text { Países más pobres } \\
\text { (menos de } \$ 200 \text { de PNB per cápita) }\end{array}$ & 130 & 150 & $160-$ & $180-$ \\
& & & 180 & 230 \\
Paises en desarrollo de ingresos medios & 630 & 950 & $1,130-$ & $1,510-$ \\
(más de $\$ 200$ de PNB per cápita) & & & 1,350 & 2,400 \\
Paj_es desarrollados & 4,200 & 5,500 & $6,700-$ & $9,000-$ \\
& & & 8,100 & 14,600 \\
\hline
\end{tabular}

Estas proyecciones inferiores son las que muchos consideran como las más probables en ausencia de grandes cambios en las políticas económicas globales. Si las estimaciones más bajas tuvieran alguna probabilidad de cumplirse, ambos grupos de países se jugarian en el mejoramiento de la cooperación.

Ello podría significar la diferencia para el equilibrio de este siglo, entre el progreso global y la prosperidad; un futuro inseguro e incómodo para los países ricos $y$ unas perspectivas cada rez más sombrías para el mundo en desarrollo.

La mayoría de los observadores están de acuerdo en que los modelos y las tasas de crecimiento de las economías industrializadas son importantes para los países pobres, ya que repercuten en la capacidad de los mismos para ejecutar sus planes de desarrollo ${ }^{1}$. Las pers-

1 Un análisis cuantitativo reciente de William Gline, de la Brookings Institution, subraya la importancia para los paises en desarrollo de las polfticas macrocconómicas de los países desarrollados. Cline llega a la conclusión de que, en igualdad de condiciones, la economía mundial operará más eficientemente si el crecimiento de los países en desarrollo sigue una tasa estable, pero sostenida, más que un crecimiento inicial rápido seguido de estagnación, con objeto de reducir la inflación.

Las ganancias adicionales de las exportaciones obtenidas por los paises en desarrollo en la primera parte del ciclo, probablemente serán menores que las ganancias de exportación sacrificadas durante la última fase.

Sus conclusiones se derivan de un análisis cuantitativo que muestra que un crecimiento económico estable, con una tasa de paro tal, que haga que la tasa de inflación no se acelere, permite las máximas ganancias del producto nacional bruto para los paises en desarrollo. Por consiguiente, es muy importante pa- 
pectivas de un mejor acceso a los mercados, niveles cle ay'uda superiores y aumento de la financiación del desarrollo no son buenas sin el crecimiento de los países ricos. Con todo, muchos comentaristas descuidan completamente la posibilidad de que el crecimiento económico continuo del mundo industrializado pueda también verse muy afectado por el progreso económico (o la falta cle éste) del mundo en desarrollo. En otras palabras, no sólo los países en clesarrollo dependen del crecimiento de los paises industrializados, sino que ahora podria quedar claro que el progreso de los páses pobres repercute en ei crecimiento económico y los niveles de empleo. no sólo porque estimula la demanda cle productos cle los pafses indus. triales, sino también porque alivia mucho las presiones inflacionistas, que son siempre el freno principal de las medidas adoptadas para reactivar el crecimiento del Nortc. Por esa razón, vale la pena considerar si las politicas que son de beneficio mutuo para los paises industrializados y en desarrollo tendrán importancia creciente para todos y si se puede o no volver a proyectar el actual orden económico, para que opere más fructíferamente tanto al servicio del Norte como del Sur.

Este documento parte de la premisa de que las economías del Norte y del Sur están más estrechamente unidas que nunca. El término abreviado para describir estas vinculaciones -interdependencia global- se lia convertido en un tópico.

A pesar de que lo que estos vínculos engloban es que el alcance de las metas económicals internas en los países desarrollados dependerá en mucho mayor medida que hasta ahora clel crecimiento y la prosperidad de los países en desarrolio; no obstante, casi no ha penetrado en la conciencia o la toma de decisiones de los políticos y' planificadores, tanto de los gobiernos de los paises desarrollados como de las organizaciones privadas.

DOS OPINIONES SOBRE LAS PERSPECTIVAS DE GREGIMIENTO DE LOS PAISES INDUSTRIALIZADOS

Existe una diferencia considerable de opinión respecto de las perspectivas de crecimiento futuro en los países industriales, después del decenio próximo.

ra los países en desarrollo que los paíces industriales crezcan con la mayor rapidez posible sin acelerar la inflación, pero que no excedan de este ritmo, im. poniendo a sus cconomías modelos de rcgulación de la demanda global de "fre. no $y$ aceleración" que rindan un crecimiento medio menor. William $R$. Cline, The Influence of Macroeconomic Policy in Industrial Countries Upon LDC Growth", Brookings Inflation Proyect Working Paper, 1977. 
Algunos analistas son del parecer que los acontecimientos que sacudieron a la economía mundial después de 1974 fueron aberrantes y que bastará aplicar una adecuada combinación de políticas económicas normales para que los países del Norte alcancen nuevamente un alto crecimiento, precios estables $y$ un bajo indice de paro.

Otros observadores no son tan optimistas. Sostienen que la economía mundial entró en una época de cambio estructural a finales de los años 60 y principios de los 70 y que las tasas precedentes de crecimiento económico no se volverán a alcanzar, a no ser que se ela. boren una serie de políticas, instituciones y criterios nuevos. El primero y quizás el más conocido ejemplo de esta escuela de pensamiento es el estudio Los limites del crecimiento ${ }^{2}$. Las proyecciones especificas de este estudio suelen desestimarse, pero sin embargo reina un acuerdo creciente en que los acontecimientos de los últimos años no son fenómenos anormales que desaparecerán, sino más bien señalan una serie de cambios fundamentales en la economía mundial. Tales eran las conclusiones de La humanidad en la encrucija$d a^{3}$, de Mihajlo Mesarovic y Eduard Pestel y clel informe más reciente del Club de Roma, La reestructuración del orden internacional 4 . Un informe actual del GATT llega a la conclusión de que la época siguiente al final de los años 60 ha sido:

"... una encrucijada histórica, en la cual las tendencias de crecimiento largo tiempo establecidas empezaron a cambiar y una dificultad general de reajuste comenzó a dejarse sentir en las economías industriales avanzadas" 5 .

Otros observadores procedentes del mundo comercial y bancario han llegado a conclusiones similares. Walter Hodley, Vicepresidente Ejecutivo y Economista Jefe del Bank of America, el mayor banco estadounidense, ha identificado algunos de los mayores cambios estructurales actualmente en curso en la economia internacional: l) crecimiento real más lento, volviendo a las tasas históricas más bajas; 2) inflación persistente (y mayor indización para amortiguar la repercusión), más un temor creciente a ulteriores pérdidas del poder adquisitivo; 3) expectativas de una vida mucho mejor con menor esfuerzo; 4) presiones masivas para una mayor equidad - de hecho,

2 Donella H., Mradows, D. Meadows, J. Randers, W. Behrens, the limits to Growth: a report for the Club of Rome on the Predicament of Mankind; Universe Books, N. York, 1972.

${ }^{3}$ Minajlo Mesarovic and Pestel, Mankind at the Turning Point the Second Report to the Club of Rome, Dutton and Co., Inc., Reader's Digest Press, Nueva York, 1974.

- Jan Tinergen, Reshaping the Intemational Order; $A$ Report to the Glub of Rome, Dutton Co., Inc., Nucva York, 1976.

- Richard Brackinurst, Trade Liberalization, Protectionism and Interdependence. Gatt Studies International Trade, Nr5, Ginebra, Nov. 1977. 
en pro del igualitarismo, causando tensiones Norte-Sur asi como urbano-rurales. Hodley llega a la conclusión que:

"... la mayoría de las viejas normas después de la segunda guerra mundial -económicas, politicas, sociales y psicológicas- se han ido para no volver y el mero desearlas no nos las devolverá".

El peso de la evidencia está cacla vez más con aquellos analistas que estiman que ha habido una serie de cambios estructurales en la economía internacional. Por lo tanto, en ausencia de nuevas políticas, tanto los países ricos como los pobres se enfrentan ahora con la posibilidad de un crecimiento más lento (con las tensiones resultantes y la incertidumbre entre $y^{\prime}$ dentro de las naciones), restricciones constantes de las inversiones que tanto se necesitan (para la producción de materias primas y mercancías manufacturadas), y precios en alza (que traerán consigo serias consecuencias para todos los países, pero especialmente para los grupos más pobres dentro de los paises).

Aquellos que pretenden que se necesitarán reajustes de gran alcance para hacer frente a las condiciones cambiadas, señalan el informe reciente del personal de la $\mathrm{OCDE}$, que pronostica que las tasas de crecimiento no alcanzarán la meta del 5 por ciento fijada por los jefes de gobierno de la oCDE recién el pasaclo mes de junio. El informe predice que, a menos que se adopten ciertas medidas expansionistas, será improbable que las tasas de crecimiento de los paises de la OCDE superen el 3 ó el 4 por ciento ${ }^{6}$.

El problema consiste ahora en que los países desarrollados y en desarrollo puedan crear un nuevo conjunto de relaciones económicas internacionales que les beneficien mutuamente, permitiendo a ambas partes alcanzar las metas económicas y sociales respectivas.

Un informe preparado para la Conferencia de las Naciones Unidas sobre Comercio y Desarrollo (UNCTAD) por economistas de la Universiclad de Pennsylvania ${ }^{7}$ apoya la aserción de que el crecimiento de los paises en desarrollo afectará al bienestar de los desarrollados. Este informe concluye afirmando que un aumento de 3 puntos porcentuales en las tasas de crecimiento de los países en desarrollo no procluctores de petróleo daría por resultado un aumento de 1 por ciento de las tasas de crecimiento de los países de la ocDe. Una mejora del I por ciento de las tasas de crecimiento de los países de la ocDE aumentaría el IPNB de los mismos en unos 45.000 millones de clólares anuales y crearía muchísimos pucstos de trabajo. En cinco años este incremento podría significar una ganancia de al menos $225.000 \mathrm{mi}$ -

' OECD Economic Outlook, Organization for Economic Cooperation and Development, № 22, Parts, diciembre 1977.

7 Trade Prospects and Capital Needs of Developing Countries, 1976-1980", 15 de abril de 1976, Conferencia de las Naciones Unidas sobre Comercio y Desarrollo. 
llones de dólares en el PNB y un incremento igualmente grande de empleos. Las ganancias secundarias serían todavia mayores.

Estas conclusiones se basan en modelos econométricos y no son definitivas. Un estudio del efecto de las acciones de los paises en desarrollo sobre las economías industrializadas durante las recesiones de 1974: y 1975, confirma la conclusión general de que los países en desarrollo son importantes para el bienestar económico de los paises desarrollados. La buena disposición de los paises en desarrollo de continuar financiando un alto nivel de importaciones de paises industriales, acudiendo a fondos prestados de fuentes oficiales $y$ privadas y utilizando las reservas tiene ... "una repercusión perceptible sobre las tendencias comerciales de los países desarrollados. El déficit de sus balanzas de pagos ha sostenido la demanda tanto como, digamos, una vigorosa expansión de la demanda alemana" 8.

Los problemas del comercio de productos básicos, alimentación, energía y población que se examinan a continuación, constituyen algunas de las razones del interés de los países desarrollados por el crecimiento $y$ prosperidad de los paises en clesarrollo. La mayoría de los estudios que se examinan se inspiran en las relaciones de los EE.UU. con el Tercer Mundo. Los argumentos se aplican, sin embargo, a los problemas más amplios de los países desarrollados. Puede haber problemas específicos de importancia mayor o menor para cada país, pero el principio de que el continuo bienestar económico y político del mundo desarrollado depende en gran medida del progreso de los países pobres hacia sus propias metas de desarrollo, es váliclo para todos.

GANANCIAS A CORTO PLAZO DE LOS SISTEMAS MUNDIALES MUTUAMENTE BENEFIGIOSOS

\section{Comercio}

No hay duda de que la liberalización comercial beneficiará a los países en desarrollo (aunque los beneficios puedan ser distribuidos en forma desigual entre los paises). Ciertamente, si los países ricos no reducen sus barreras comerciales, las perspectivas de crecimiento de los paises en desarrollo disminuirán considerablemente.

Los países en desarrollo han conseguido un éxito notable al incrementar las exportaciones de sus productos manufacturados en los dos últimos decenios. Dichas exportaciones crecieron a una tasa de

Johin Attolsen and Jean L. Waelbroeck: "The Less Developed Countries and the International Monetary Mechanism", American Economic Review, mayo 1976, Vol. 66, No 2. 
cerca de un 16 por ciento anual desde 1960 hasta 1974. Las importaciones de productos manufacturados de los EE.Uu., procedentes de los países en desarrollo, siguieron la misma pauta, aumentando diez veces de 1960 a 1974, pasando de 844 millones de clólares a 9.900 millones de dólares (dólares EE.uU. constantes).

Su tasa anual de crecimiento fue de cerca del 18 por ciento. Estas ganancias se obtuvieron a pesar de que las barreras comerciales a los productos manufacturados de los paises en desarrollo no experimentaron rebajas importantes durante la liberalización comercial de los años 60.

Las barreras comerciales reduciclas mejoran claramente la situación económica de los países en desarrollo, pero entre muchos grupos afectados de los países industriales existe una oposición creciente al costo actual del aumento del comercio con los países en desarrollo (que resulta de la liberalización comercial). Esta oposición, a su vez, ha conducido a ejercer presiones crecientes para que se adopten una variedad de medidas proteccion:stas.

Sin embargo, el cuadro siguiente mucstra que, en términos generales, los países en desarrollo no han sido la causa de los actuales deficit comerciales o de la pérdida de empleos en los países desarroIlados, aunque haya habido algunas industrias afectadas seriamente por la concurrencia de las importaciones.

Los países en desarrollo compran más mercancías y servicios norteamericanos que muchos otros paises y zonas que se cuentan tradicionalmente entre los mercados mayores para los productos estadounidenses. De hecho, durante lat major parte cle los últimos años, las exportaciones norteamericanas a los paises en desarrollo crecieron a una tasa más rápida que las exportaciones a los países desarrollados.

En el período que va de 1970 a 1975 , las exportaciones de los EE.UU. a los países en desarrollo que no forman parte de la opeP experimentaron un alza de más del 19 por ciento al año, en comparación con la tasa anual media de crecimiento de otros paises desarrollados, que registraron el 15,5 por ciento en el mismo espacio de tiempo. (Para algunos de los países en desarrollo de industrialización rápicla, el aumento de lá tasa media anual fue superior al 50 por ciento.) Estas exportaciones no sólo beneficiaron a empresas del sector exportador, sino que también proporcionaron trabajo a muchos estadouniclenses. Hiay buenas posibilidades cle crecimiento continuo de las exportaciones de los EE.uv., ya que la demanda de los países en desarrollo está lejos de haberse saturado y podría extenderse con rapidez a medida que dichos paises avancen económicamente. Si el poder adquisitivo de los países en desarrolio se mantiene (y preferiblemente se amplie), es probable que el conercio en- 
tre el Norte y el Sur se incremente a una tasa mucho más rápida que la del comercio entre los paises industrializados.

Pero el comercio con los países en desarrollo es una calle de doble dirección y a menos que los paises en desarrollo puedan exportar a los Estados Unidos y a otros países industrializados, no podrán obtener las divisas necesarias para comprar, a su vez, los productos manufacturados que se producen en los países industriales.

EL COMERCIO DE MANUFACTURAS DEL MUNDO RICO CON LOS PAISES POBRES

miles de millones de $\$$

\begin{tabular}{|c|c|c|c|c|c|c|c|c|c|}
\hline & \multicolumn{3}{|c|}{$E E . U U$} & \multicolumn{3}{|c|}{$C E E$} & \multicolumn{2}{|c|}{$J A P O N$} & \multirow[b]{2}{*}{ Bal: } \\
\hline & Exp. & $\operatorname{Imp} . *$ & Bal. & Exp. & $I m p$ & Bal. & $\operatorname{Exp}$. & $I m p$. & \\
\hline 1972 & & & & & & & & & \\
\hline $\begin{array}{l}\text { eMD** } \\
\text { con petróleo }\end{array}$ & 1.96 & 0.05 & 1.91 & 5.03 & 0.32 & 4.71 & 1.78 & 0.02 & $1: 76$ \\
\hline $\begin{array}{l}\text { PMD } \\
\text { sin petróleo }\end{array}$ & 7.61 & 6.13 & 1.48 & 12.79 & 4.23 & 8.56 & 7.14 & 1.19 & 5.95 \\
\hline Total PMD & 9.57 & 6.18 & 3.39 & 17.89 & 4.55 & 13,27 & 8.92 & 1.21 & 7.71 \\
\hline $\begin{array}{l}1976 \\
\text { PMD }\end{array}$ & & & & & & & & & \\
\hline con petróleo & 9.95 & 0.14 & 9.81 & 22.28 & 0.62 & 22.66 & 9.01 & 0.08 & 3.93 \\
\hline $\begin{array}{l}\text { PMD } \\
\text { sin petróleo }\end{array}$ & 18.47 & 14.82 & 3.65 & 25.70 & 11.05 & 14.65 & 16.38 & 3.52 & 12.86 \\
\hline Total PMD & 28.42 & 14.96 & 13.46 & 48.98 & 11.67 & 37.31 & 25.39 & 3.60 & 21.79 \\
\hline
\end{tabular}

* El comercio nortcamericano se indica FOB-FOB. Sus importaciones se han incrementado aqui en un 6 por ciento para ponerlas, aproximadamente, en los términos FOb-CrF cmpleados por la CEE $y$ el Japón.

Fuente: catr, sobre la base de cifras de las Nu. (The Economist, 31 de diciembre de 1977).

** PMD $=$ Países menos desarrollados.

Las tendencias prevalecientes desde 1975 evidencian en forma más bien dramática que existe una interrelación directa entre el progreso de los países en desarrollo, su capacidad de comprar mercancías producidas en los países desarrollados y' su repercusión en el empleo y en el crecimiento económico del mundo industrial.

La alta tasa de crecimiento de las exportaciones de los EE.UU. a 
los países en desarrollo se redujo mucho durante 1976 y los 10 primeros meses de 1977. Así vemos que, mientras que las exportaciones de los EE.UU. a los paises desarrollados crecieron en un 9 por ciento, las exportaciones a la OPEP disminuyeron cerca de un 3 por ciento (en dólares) entre 1975 y 1976.

La desaceleración del ritmo de compras a los Estados Unidos coincide con una fase en la cual los paises en desarrollo han sufrido una serie de reveses en sus economias, originados en la recesión de los países industriales y' la inflación planetaria, la cual ha aumentado drásticamente los precios que dichos paises han de pagar por la energía, la alimentación y los productos manufacturados de importación.

Como resultado del mayor costo de las importaciones y de la disminución de las exportaciones (con un incremento resultante de la carga de la deuda), estos países han aminorado sus importaciones clesde los países industrializados.

De hecho, la disminución de las importaciones de productos norteamericanos por los países en desarrollo no pertenecientes a la OPEP, ha contribuido al cléficit comercial de los Estados Unidos. Las estimaciones de la OCDE indican que de los 40.000 millones de dólares de salcio negativo que arroja la balanza comercial de los EE.uU. entre 1975 y $1977,15.000$ millones se han producido en el comercio con los países en desarrollo no pertenecientes a la OPEP 9 .

Está claro que la capacidad de los paises en desarrollo para comprar mercaderías produciclas por los países industriales tendrá una repercusión importante en el progreso económico de ambos grupos de paises.

Cercenar el acceso de los países en desarrollo a los mercados de los países desarrollados, va a revelarse por último como algo autodestructivo, ya que ignora los'beneficios que se derivarian de la liberalización del comercio para los países desarrollados. Los beneficios del aumento del comercio son triples: el comercio proporciona empleos, reduce la inflación y mejora la competitividad a largo plazo de la economía.

Un simple cálculo indica que si los paises desarrollados fueran a crecer en el decenio próximo aproximadamente con la misma tasa que en los años $60-y$ si la parte de los Estados Unidos en las importaciones de los países en desarrollo fuera la misma que en el decenio último- podría preverse que los países en desarrollo importarian otros 27.000 millones de dólares más de mercancías de los Estados Unidos por año, para 1985. Utilizando proyecciones normales, este incremento podría suponer tanto como dos millones más de empleos en las industrias norteamericanas de exportación, lo que tiene

" ocDE, Economic Outlook, número 22, p. 8, París. 
como contrapartida el número de posibilidacles de trabajo que podrían perderse en caso de una liberalización comercial.

Un estudio próximo a publicarse, del Overseas Development Council (Consejo de Desarrollo de Ultramar), estima que si el comercio textil se excluye de la liberalización, las reducciones arancelarias del 60 por ciento de los productos exportaclos por los países en desarrollo requerirán sólo pequeños reajustes laborales ${ }^{10}$.

El estudio estima que quizás 31.500 empleos se perderían como resultado del aumento de las importaciones de los EE.uU. procedentes de los paises en desarrollo. Sin embargo, dicho pais podría ganar más de 20.000 empleos como resultado directo del incremento de las exportaciones, debido al reciclaje de las ganancias de exportación obtenidas por los países en desarrollo. El resultado neto arrojaría la pérdida de unos 11.000 empleos. El total es igual a cerca de una décima parte del uno por ciento del total de la fuerza de trabajo en Estados Unidos en 1976.

El hecho de que las ganancias totales para la economía superen con creces a las pérdidas de empleos directamente atribuibles al incremento de importaciones de los países en desarrollo, no debe paliar la importancia de los programas de asistencia al reajuste, con especial atención a las industrias seriamente afectadas, como el calzado y el cuero.

Un estudio en preparación de la Brookings Institution estima que los costos de un programa de asistencia a la reestructuración ascenderían sólo a 600 millones de dólares para los trabajadores y empresas afectadas por las importaciones de todos los paises ${ }^{11}$.

Tanto por razones políticas como morales, la liberalización comercial no debe ser un sector en que unos pocos paguen las ganancias de muchos. Por lo tanto, un objetivo prioritario para los países desarrollados debe ser un aumento de los beneficios y una mayor liberalización de los programas actuales de asistencia, al igual que planificación anticipada para facilitar la transición de algunos tipos cle industrias a otros.

Las exportaciones a los países en desarrollo estimulan a la vez el crecimiento y' el empleo del país, mientras que las importaciones de los mismos paises tienen una repercusión favorable sobre la inflación.

El estudio de la Brookings Institution indica que una reducción a escala mundial del 60 por ciento de los aranceles actualés, reduciría los precios de las importaciones lo suficiente para rebajar el Indice de Precios al Consumidor de los Estados Unidos en una cuarta

10 Thomas Brrneerg, Trade Relations Between Rich and Poor Counlries, Overseas Development Council, Washington D.C.

11 WILLLM R. CaINE, et al. Trade, Welfare, and Employment Effects of MIUltilateral Trade Negotiations in the Tokio Round. The Brookings Institution, Washington, D.C. 
parte del uno por ciento, aproximadamente, lo que no es poco, dada la gran preocupación que reina en el país ante cualquier alza de precios. Además, las importaciones más baratas sirven también para reducir la inflación, pues hacen la competencia directa a los productores locales y aumentan así la eficiencia nacional.

Pero los efectos secundarios pueden ser mucho mayores. E1 mismo estudio llega a la conclusión de que las ganancias sociales en los Estados Unidos, resultantes del paro evitado gracias a la repercusión antiinflacionista, podrian elevarse a 14.000 millones anuales, haciendo ascender los beneficios sociales totales, derivados cle efectos más convencionales del libre comercio - tales como economías de escala, alicientes a la competitividad y aumento de la eficiencia- hasta en un tercio.

Estimular la demanda del mundo en desarrollo para las exportaciones de los países industriales puede ser también una forma no inflacionista de restaurar el crecimiento dentro de las economías de la ocDE. Si es verdad que las presiones inflacionistas obstaculizarán cada vez más la adopción de políticas que aceleren el crecimiento y disminuyan el desempleo en los países industrializados, entonces será muy recomendable aplicar una política macroeconómica, que estimule las importaciones por parte de los países en clesarrollo, de los productos de los paises industriales.

Tal política incrementará la demanda de mercaderías de los países industrializados, sin el mismo efecto directo inflacionista del consumo interno adicional o del aumento de los gastos gubernamentales clebidos a las políticas liscales expansionistas.

E1 Comisario de las Comunidades Europeas, Claude Cheysson, y un informe del Comité del Presupuesto del Senado de los EE.vu. han sugerido, cada uno por su lado, que el método mejor y menos inflacionista de recuperación de los países OECD sería estimular la demanda de importaciones por parte de los paises en desarrollo. Según el informe del Senado "... Mayor demanda exterior significa que se requerirá menos en el interior. El aumento de las importaciones de las economias desarrolladas y de otros patses deudores reclucirá también la necesidad cle estimulos fiscales y déficit presupuestario en los Estados Unidos" 1‥

Un ejecutivo de la Singer Company sacó a colación un tema similar al escribir que el aumento del crecimiento económico de los paises en desarrollo podría “... servir para fomentar las exportaciones de las naciones industrializadas. $Y$ estas mayores exportaciones

12 "The International Economy and the Federal Budget", preparado por el Comité del Presupuesto, Senado de los Estados Unidos, 30 de diciembre de 1976, p. 12. 
podrían a su vez tener un efecto multiplicador en la inversión, el crecimiento, e incluso las exportaciones norteamericanas".

Finalmente, quienes desean restringir el crecimiento del comercio cntre los países industriales $y$ los países en desarrollo ignoran el hecho de que los factores básicos de la producción internacional pueden estar en un proceso de cambio.

Como concluye un informe reciente del Banco Mundial: "El modelo internacional de ventajas relativas está cambiando rápidamente y los paises en desarrollo se están convirtiendo en proveedores de una amplia gama de manufacturas. Simultáneamente, la producción se estanca y el empleo disminuye en los sectores correspondientes de los países industrializados. Este es el proceso que hace esperar la teoría y la historia económica, pero se está realizando a velocidad sin precedentes, por lo que no tiene nada de extraño que esta vasta reestructuración de la economía mundial cree fricciones y suscite.respuestas defensivas. Es evidente que existe el potencial para una expansión considerable de la especialización internacional, aunque el ritmo de aprovechamiento de las oportunidacles será determinado en gran medida por las políticas económicas, tanto cle los países in"dustrializados como de los paises de nueva industrialización" 13.

Si esta conclusión es correcta, los países industriales tendrán que operar los cambios necestrios para absorber las exportaciones crecientes de los países en desarrollo, no sólo para sostener la demanda de sus propias exportaciones y reducir la inflación, sino también para mantener a largo plazo las ventajas relativas de sus posiciones económicas.

\section{Los productos básicos}

Los precios de diversos productos y materias primas han sido la gran preocupación de los países ricos desde las alzas de comienzos del 70 $y$ de los países pobres por un período considerablemente más largo.

Ambos bandos han llegado a comprender que los acuerclos efectivos sobre productos básicos reportarán beneficios substanciales a los países, tanto industriales como en desarrollo.

$\therefore$ Si se proyectan convenientemente, los acuerdos sobre estabilización de precios prevendrían igualmente las fluctuaciones excesivas de éstos y darían la certidumbre que estimularía el incremento de las inpersiones y la explotación de nuevas fuentes de materias primas (así como la explotación a fondo de las fuentes existentes).

. Funcionarios de los países desarrollados reconocen ahora que mientras los incrementos en los precios de los productos básicos tien-

1s Prospects for the Developing Countries, 1978-1985, Personal de Política de Desarrollo, Banco Mundial, noviembre 1977, p. 44. 
den a producir un aumento general de los precios, la flexión subsiguiente de dichos precios no se refleja completamente (por un sinnúmero de razones) en el precio final de los productos terminados.

Este es el llamado "efecto del trinquete", que quiere decir que aunque los precios suelen subir, raramente bajan. Como resultado, se institucionaliza la inflación.

Asimismo, los países desarrollados preocupan por los precios de los productos básicos a causa de: 1) que un rápido incremento de los precios de los productos básicos puede motivar pérdidas importantes en la producción y el empleo; 2) que fluctuaciones amplias de los precios de los productos búsicos suelen originar una falta de inversión en nuevas capacidades en las fases primarias y de producción.

Sin tales inversiones, la oferta no puede satisfacer la exigencia de una reactivación del crecimiento en años sucesivos, presionando los precios más hacia el alza.

Un análisis recicnte simula lo que hubiera podido ocurrir si un grupo de ocho productos básicos "capitales" (café, coco, té, caucho, yute, sisal, cobre y' estaño) y otros cinco productos (trigo, arroz, lana, bauxita $y$ mineral de hierro) hubieran estado amparados por acuerdos de estabilización de precios durante el periodo 1963-1972 ${ }^{14}$.

lis estudio concluye que las entradas de los paises en desarrollo habrian experimentado un alza de cinco mil millones de dólares en valor descontado actual durante todo el decenio.

Las ganancias económicas para los Estados Unidos tan sólo del paro que se logró evitar y la pérdida del $\mathrm{PNB}$ que se salvó, que habrian resultado de la reclucción de las presiones inflacionistas, hubieran ascendiclo a 15.000 millones de dólares en el decenio. Además, tanto los países clesarrollados como los paises en desarrollo se beneficiarian de la menor incertidumbre en la planificación, las tasas más altas de inversión para los productores a causa de la disminución del riesgo y la ampliación de la oferta para los consumidores a consecuencia del aumento de la inversión.

Otro estudio patrocinado por la 'ocDe muestra que la ganancia social neta, en un lapso de 20 años, de una reserva de estabilización de 15 millones de toneladas de trigo, tanto por las repercusiones in. ternas como por el comercio internacional, se elevaria a $2.500 \mathrm{mi}-$ llones de dólares ${ }^{15}$.

Esta cifra modesta no tiene en cuenta ninguna ganancia en la producción macroeconómica, que podría lograrse gracias a la reduc-

14 JERE R, BERHan, International Commodity Agreements: An Evaluation of the uNcTad Integraled Commodity Programme. Series de monografía número 9. Overseas Development Council, NIED,

is Lance TaYlor, et al, Grain Reserves, Emergency Relief and Food Aid. Overseas Development Council, NIED. 
ción de las dificultades en el control de la inflación y el incremento resultante de empleos.

Por ejemplo, una reserva alimentaria global evitaría la repetición de la experiencia del 1973-1974, cuando una insuficiencia de un tres por ciento de la producción del grano produjo un incremento de más del 300 por ciento de los precios, aumento que contribuyó a la inflación planetaria.

Finalmente, el tiempo requerido para incrementar la producción de muchas materias primas es cada vez mayor y' a no ser que se adopten medidas en el futuro próximo para aumentar las inversio. nes en aquellas industrias, es muy probable que se operen alzas espectaculares de precios en los años 80. Ello da la preferencia no sólo a los acuerdos sobre reservas de estabilización, sino también a los acuerdos que brindan seguridades contra los riesgos políticos.

La propuesta estadounidense cle crear un Banco Internacional de Recursos, cuya base sea multilateral, podría facilitar la seguridad financiera y política para garantizar la inversión continua en nuevas exploraciones y producciones.

\section{FINANCIAGION DEL DESARROLLO Y ENDEUDAMIENTO}

El gran aumento del endeudamiento de los países en desarrollo y la necesidad resultante de continuar el crecimiento y la expansión de sus exportaciones, para hacer frente al desembolso de la deuda, es otra razón de por qué la relación entre el crecimiento y el progreso de los países en desarrollo y desarrollados ha adquirido tanta importancia en los últimos cinco años.

Los países en desarrollo, no exportadores de petróleo, acumularon una deuda pública global (incluyendo la no desembolsada) que creció en un 84 por ciento entre 1972 y 1976 , alcanzando un nivel estimado de cerca de 171.000 millones de dólares a finales de 1976.

La deuda privada no garantizada por los Gobiernos de los países en desarrollo podría añadir otros 25-30.000 millones a aquella cantidad, lo que elevaría la deuda total de dichos países a unos 200.000 millones de dólares.

La carga de la deuda recae desigualmente sobre varios países; gran parte de ella, especialmente la contraída con los bancos privados, recae en los países en desarrollo de ingresos medios. Para algunos países, los pagos del servicio de la deuda absorben más del 20 por ciento de las ganancias obtenidas con sus exportaciones.

Los analistas difieren en sus puntos de vista sobre la seriedad del problema mundial del endeudamiento.

Los pesimistas opinan que, a causa de la subida de los precios del petróleo y de los productos manufacturados, el endeudamiento 
de los países en desarrollo crecerá hasta tal punto que tal vez les sea imposible pagar sus deudas.

Las instituciones financieras privadas de los países desarrollados, especialmente en los Estados Unidos, se enfrentarán con el dilema de prestar más, con graves riesgos, o correr el peligro de que Ios mayores países deudores no paguen ${ }^{16}$.

La opinión optimista sostiene que la deuda de los países en desarrollo no es demasiado grande si se tienen en cuenta el crecimiento de la exportación y de la inflación, y que los principales prestatarios se las arreglarán para pagar sus deudas en pocos años.

El Banco Mundial y el Fondo Monetario Internacional mantienen este punto de vista, que también es compartido por un estudio reciente de la OCDE sobre el problema del endeudamiento ${ }^{17}$.

La opinión optimista parece tener razón en este momento, pero es probable que se llegue a una encrucijada peligrosa cuando finalice el decenio presente, cuando los numerosos préstamos a corto plazo aceptados por los países en desarrollo en la época de la crisis del petróleo empiecen a vencer.

El estudio de la oCDE señala que los peligros potenciales a largo plazo son: 1) el aumento de la carga del servicio de la deuda proyectado para el año 1982; 2) la posibilidad de que los bancos comerciales no sean capaces de ofrecer nuevos préstamos con los tipos de intereses previos o estimen prudente reducir sus riesgos en algunos países en desarrollo, por lo menos, y 3) la posibilidad creciente de que los paises industrializados sólo puedan activar un crecimiento lento o incluso recaer en la recesión - una eventualidad que agigantaria sobremanera el problema del endeudamiento.

Los costos de una serie de incumplimientos de los principales países en desarrollo para las economías industrializadas no pueden calcularse con exactitud. Es más, las estimaciones varian mucho, sean cuales fueren los puntos de vista de cada uno. Sin embargo, está claro que la continuidad del bienestar financiero de los países en desarrollo será un factor importante en la estabilidad del sistema financiero internacional y como tal, de primordial importancia para los Estados Unidos y otras economias industriales.

El sector bancario tiene que jugar un papel importante en el bienestar financiero de los países en desarrollo.

Aunque se han exagerado los riesgos que corren los bancos comerciales en el mundo en desarrollo, la impresión que causaría el

${ }^{16}$ International Debt, the Banks, and U. S. Foreign Policy, Informe dal. personal, Comité de Relaciones Exteriores, Subcomité de polftica económica extranjera, 1977 .

1* Gondon W. Sarith, The External Debt Prospects of the Non-Oil Exporting Countries: An Econometric Analysis. Series de monografia, número 10. Overseas Development Council NIED. 
incumplimiento de un importante pais en desarrollo debido a la incapacidad de cubrir el servicio de los préstamos pasados o reunir los requisitos para obtener créditos adicionales sería considerable, incluso tan sólo desde el punto de vista psicológico.

Por lo tanto, todas las partes tienen interés en mantener la capacidad del servicio de la deuda de los países en desarrollo, y en que éstos puedan seguir obteniendo nuevos créditos a fin de recobrar y/o mantener su ritmo de desarrollo.

\section{LAS VENTAJAS A LARGO PPLAZO DE LOS SISTEMAS GLOBALES DE BENEFICYO MUTUO}

En la sección precedente se analizaron algunas de las ventajas de una serie de sistemas globales de mayor eficiencia y mutuo beneficio, que pueden revertir en los países desarrollados en el próximo decenio. A larzo plazo - esto es, hasta el final de siglo- la colaboración recíproca entre países ricos y pobres en una serie de problemas globales del sistema será también necesaria.

Las posibilidades de obtener ganancias mutuas como fruto de la eficiencia global son considerables en tres zonas cruciales: alimentación, energia y población.

Los países ricos se enfrentarán con costos cada vez mayores -tanto directos como indirectos- si no respaldan los esfuerzos de los países en desarrollo para incrementar la producción, desarrollar nuevas fuentes de energía y crear los tipos de programas de desarrollo que son indispensables para estabilizar la población.

En cada caso, la acción conjunta en estos problemas importantes proporcionará ganancias a los países en desarrollo y contribuirá a sus esfuerzos para satisfacer las necesidades de sus poblaciones.

Alimentación.-- La "crisis mundial" adquirió importancia para los países ricos en 1973 cuando, por toda una serie de razones, las cosechas fueron muy malas en el mundo.

El aumento resultante de la demanda de alimentos en el mercado estadounidense provocó el aúmento de los precios para los consumidores y los productores.

Para mucha gente del mundo en desarrollo, sin embargo, la "crisis mundial" forma parte permanente de la realidad. Los costos astronómicos de las importaciones de alimentos, combustibles, fertilizantes y productos industriales después de 1974, exacerbaron - ya que no crearon- el problema de los países en desarrollo.

En ambos bandos, ricos y pobres, los acontecimientos de 1974 sirvieron como catalizador para una reevaluación más exacta de có- 
mo se pueden satisfacer las necesidades alimentarias de los paises desarrollados y en desarrollo a finales de siglo ${ }^{18}$.

La mayoría de los análisis llegaban a la conclusión de que la producción alimentaria de los países en desarrollo tiene que incrementarse drásticamente si se quiere que estos países atiendan a las necesidades nutritivas de sus poblaciones crecientes y que los productores de los paises desarrollados (y especialmente de los Estados Unidos) contengan los costos encumbrados de la alimentación, que ha sido un factor tan importante de la inflación actual.

En la época 1973-1974, por ejemplo, los precios alimentarios crecientes contribuyeron tanto a la inflación de los Estados Unidos y a la global como los costos ascendentes del petróleo.

El argumento en favor del aumento de la producción de los países en desarrollo para el común beneficio de los paises inclustriales del hemisferio norte y los paises en clesarrollo del sur, estriba en los hechos siguientes: en el cuarto de siglo pasado ha surgido un desequilibrio global creciente de la producción y el consumo alimentario. Los paises en desarrollo, los cuales eran virtualmente autosuficientes en su producción alimentaria en 1950, tuvieron que importar 15 y 20 millones de toneladas de cereales en 1970, la mitad de los cuales en forma de ayuda alimentaria. En 1975, las importaciones de grano de esos países alcanzaron los 45 millones de toneladas.

Las proyecciones de la situación alimentaria para 1990 del International Food Policy Research Institute (Instituto de Investigación de Política Alimentaria Internacional) cuya sede está en Washington, muestran que los países en desarrollo necesitarán el triple de sus importaciones de grano, a menos que no se opere una mejora fundamental de su capacidad para producir alimentos ${ }^{18}$.

Estas proyecciones tienen muchas implicaciones adversas para los países en desarrollo: sus importaciones crecientes requerirán cantidades cada vez mayores de divisas que escasean; las importaciones de alimentos (más que el aumento. de la producción interior) significan mayor clesempleo y' subempleo en las zonas rurales, en un momento en que la situación laboral en ambos sectores es muy grave y en que el paro entraña muy a menudo el hambre, lo cual supone que quienes ya sufren de hambre y malnutrición sufrirán aun nás.

18 IVorld Food and Nutrition Study, preparado por, cl Comité Ejecutivo, NRG Estudio sobre alimentación mundial de la Comisión de Relaciones Internacionales, Acatemia Nacional de Ciencias, Washington, D. C., 1977. Consúltense tambićn los informes del Conscjo Mundial de Alimentación preparados para su ù11 ma reunión y Reshafing the International Order.

"Food Necds of Developing Countries: Projections of Production and Consumption to 1990 , diciembre $197 \%$. Informe de investigación 3 , International Food Policy Rescarch Institute. 
Las proyecciones actuales indican que será menester aumentos tremendos de la producción de cereales para satisfacer el incremento de la demanda en los países desarrollados. En 1990, la demanda de cereales en los paises desarrollados habrá aumentado a 846 millones de toneladas (617 millones de toneladas en 1970); este incremento de más de 200 millones de toneladas equivale aproximadamente a la producción actual de grano en los Estados Unidos.

Durante el mismo período, la demanda en los países en desarrollo de economía de mercado, aumentará en unos 350 millones de toneladas -casi una vez y media la producción actual de los EE.vu.

Con los modelos presentes de producción, bastante más de 100 millones de toneladas de este aumento de la demanda alimentaria tendrán que cubrirse con el incremento de las exportaciones de Norteamérica en 1990, con cantidades más elevadas en adelante. Norteamérica se ha convertido cada vez más en el granero del mundo, con una ampliación de las exportaciones que han pasado de $34 \mathrm{mi}$ llones de toneladas en 1960 a $\mathrm{I} 00$ millones de toneladas a mediados de los años 70.

Todas estas proyecciones tienen repercusiones importantes. Un estudio de la Academia Nacional de Ciencias ha indicado que será mucho más difícil para los Estados Unidos -el mayor exportador de cereales del mundo- y para otros países desarrollados, satisfacer las necesidades alimentarias mundiales, sin causar alzas fuertes de los precios de los cereales. Aunque no se citan cifras específicas al respecto, los incrementos de la producción y la distribución podrían ser incluso del 50 y al 100 por ciento de un decenio. La repercusión inflacionista de tales aumentos sería muy importante.

El incremento de los costos de los insumos, resultante de la necesidad de utilizar cada vez más las tierras marginales, los escasos suministros de agua y las perspectivas de disminución de los incrementos de rendimientos debidas al aumento del empleo de fertilizantes y de otros insumos de utilización intensiva de energía, es la razón de que aumenten los costos de producción.

Hasta que no haya otra generación de investigaciones básicas que aplicar, lo que llevará al menos un decenio, sólo será posible aumentar los rendimientos por hectárea a través del incremento de los insumos.

El estudio de la Academia Nacional indica que los costos de los fertilizantes aumentarán radicalmente en los años venideros, debido por lo menos a tres factores: al costo creciente de las materias primas de las que se extrae el fertilizante; a las fuertes subidas del costo de los servicios de producción de fertilizantes nitrogenados y fosfatados, y a los costos en rápido aumento del transporte y la distribución.

El costo de producción del fertilizante de urea en los países in- 
dustrializados, por ejemplo, según las proyecciones, debcria aumentar de 176 dólares en 1977 a 331 dólares en 1990 (en dólares constantes de 1977). La conclusión es obvia. Habrá una subida considerable de precios si América del Norte, el mayor suministrador de cereales del mundo, aumenta mucho su producción en los dos decenios próximos.

Por esta razón, es un hecho generalmente reconocido que la respuesta a los problemas alimentarios mundiales debe consistir prioritariamente en el incremento de la producción en los países en desarrollo.

Si los países del Sudeste Asiático pudieran superar los obstáculos financieros, tecnológicos y organizativos para aprovechar mejor el agua $y^{\prime}$ la mano de obra $y^{\prime}$ otros recursos tan infrautilizados, podrían probablemente duplicar e incluso triplicar la producción alimentaria a los niveles actuales de precios internacionales.

Lo mismo vale para la región dsl Sahel, que tiene capaciclad potencial para colmar las necesidades alimentarias de su población en aumento, e incluso para exportar alimentos a los países vecinos.

A mayor abundamiento, existe la prueba de que en los paises que cuentan con poca tierra, pero con amplias poblaciones, las explotaciones agricolas pequeñas y de trabajo intensivo pueden producir más, por hectárea, que las explotaciones mayores, siempre que estén provistas debidamente de una variedad de servicios rurales.

Al incrementar la producción alimentaria en los países en desarrollo de manera tal que los que necesitan más que nadle que aumente el abastecimiento alimentario tengan acceso a él (lo que a su vez exige más ingresos y más empleos), se obrará en interés cle los pueblos, no sólo de los países en desarrollo, sino también de los Estados Unidos y otros países desarrollados.

Sin embargo, pocos norteamericanos comprenden en la hora actual que lo que deciden los gobiernos y los dirigentes de aquellos países acerca del desarrollo rural y de la producción alimentaria en sus propios paises, tendrá una repercusión considerable en los años venideros sobre los precios que los estadounidenses pagan por Ios comestibles en los supermercados.

El granero norteamericano no puede facilitar otros 100 millones de toneladas de exportaciones para los países en desarrollo, mientras tenga que satisfacer simultáneamente la demanda creciente tanto en Norteamérica como en otros países desarrollados, sin drásticos aumentos de precios durante los años 80 y 90 , para equilibrar la oferta y la demanda.

Energia-- La situación energética global es otra zona en doncle los acontecimientos que sucedan en el mundo en desarrollo, desde ahora hasta el final clel siglo, pueden tener una repercusión importante 
y directa en el crecimiento y el progreso continuo de los países inclustriales, $y^{\prime}$ en clonde las politicas alternativas pueden beneficiar tanto a unos como a otros.

El alya drástica cle los precios del petróleo que ocurrió en 1973 clejó anticuadas todas las estimaciones existentes de la demanda de energía para el futuro, tanto para los ricos como para los pobres. A pesar de ello, pocas proyecciones hechas después de 1973 tienen suficientemente en cuenta las necesidades energéticas de los paises $\epsilon n$ desarrollo. No consideran tampoco dichas proyecciones de qué manera los modelos alternativos de desarrollo afectarán el consumo de energia por parte de los países en desarrollo, especialmente el uso del petróleo, lo cual a su vez puede tener repercusiones diferentes sobre la economía petrolera mundial.

De hecho, casi todas aquellas proyecciones dan por sentado que los países en desarrollo no incrementarán mucho el uso de la energria como resultado del mayor ritmo de modernización de sus zonas rurales en el período contemplado por las estimaciones. O, dicho con otras palabras, todos los estudios han asumido que la gente que vive en las zonas rurales de los paises en desarrollo no pasarán de las fuentes tradicionales de energía al petróleo con velocidad mayor que las registradas históricamente en esos países.

Dos hechos nos llevan a interrogarnos sobre si estas estimaciones se basan en hipótesis erróneas. Primero, la gran mayoria de los habitantes de los países en desarrollo siguen usando fuentes de energia no comerciales para la mayor parte de sus actividades; es decir, depenclen de madera, estiércol, residuos de cosecha y energía animal y humana para casi toda la energía que consumen. Pero todo grado satisfactorio de desarrollo exige un fuerte incremento en el empleo de energía no humana. Segundo, existe actualmente un creciente consenso internacional en cuanto a que los programas de desarrollo han de procurar satisfacer las necesidades humanas minimas de la mayoría de la humaniclad en un arco preciso de tiempo-digamos para finales de siglo.

Realizar esta meta, sin enbargo, exigirá considerables aumentos del uso de energía y los cálculos actuales indican j'a que los países en desarrolio consumirán -e importarán- tanto petróleo en el año 2000 como los Estados Unidos hov.

Naturalmente, es probable que las demandas en competencia impidan una tal expansión. Un resultado mucho más verosímil, si no se hace nada para iniciar un esfuerzo cooperativo global a fin de satisfacer las necesidades energéticas muncliales, es que el mayor poder adquisitivo de los países desarrollados les permitirá pagar el precio más alto de los suministros de petróleo restantes y que los países en desarrollo, por consiguiente, tendrán que reducir sus compras o usar mayores cantidades de sus escasas reservas de divisas 
para intentar mantener Ios programas de desarrollo existentes. Ello acarrearí probablemente el agotamiento acelerado de los suministros finitos cle petróleo del mundo, el aumento de la inflación $y$ la reducción del crecimiento y del clesarrollo, especialmente de los paises en clesarrollo más polsres.

Aparece aquí una colusión de trayectorias, entre la confianza en la energía basada en el petróleo para alcanzar las metals de clesarroIlo mundial $y^{\prime}$ el descenso de la producción mundial de petróleo, que la mayoria de los expertos opinan que ocurirí antes de final de siglo.

Un criterio alternativo, sin embargo, podria mostrarse beneficioso a largo plazo para ambos grupos de páses. Este enfoque vincularia la conservación clel uso energético en los países clesarrollaclos yt la asistencia a los paises en clesarollo, para adoptar estrategias energéticas que no se basen exclusivamente en el petróleo y los ayuden en la príctica a avanzal: clirectamente hacia el empleo cle las tecno. logias energéticas postpetroleras clel futuro.

Un enfoque cooperativo y global del problema energético tendería a incrementar la producción local de energía de los países en desarrollo partiendo de una diversidad de fuentes, incluysndo nuevos usos del petróleo, energía hiclroeléctrica, carbón y', parat aquellos que elijan tal senda, energía nuclear. Pero este criterio hace que, a largo plazo, los paises en clesarrollo dependan caclit vez más cle fuentes renovables cle energí, especialmente solar. Por este camino muchos paises en clesarrollo empezarán a utilizar a finales de los 80 y en los 90 las que, para la malyoría cle paises industriales, serán las tecnologias del siglo veintimo.

Los paises en desarrollo gozan de diversas ventajas naturales que les permitirán (con apoyo suficiente de los países ricos) recurrir cada vez más a fuentes renovables de energía. En su inmensa mayoria, dichos paises no cuentan con las inversiones masivas en infmestructuras energéticas basadas en el petróleo, que caracterizan a los países industrializados.

Muchos de ellos tienen más lu\% solar que los industriales, Io que hace que lat energía solar sea la forma más aptal para ellos, y sus condiciones climáticas permiten el crecimiento más rápiclo de la vegetación para leña y biogasificación. Finalmente, la índole descentralizada de la energín renovable la convierte en una fuente ideal de energía para programas de desarrollo rural en pequeña escalá.

El criterio de cooperación paral satisfacer las necesidacles energéticas mundiales ofrece una variedad de beneficios para toclos. Proporcionará a los paises en desarrollo la oportunidad de evitar inversiones costosas ( $y$ sumamente fútiles) en tecnología energética petrolera y en infraestructuras, y orientarse rápidamente hacia el uso creciente de las fuentes energéticas del futuro. 
Ambos grupos cle países tienen interés en que la investigación y el desarrollo necesarios para rebajar los costos de nuevas tecnologias se pongan en marcha lo antes posible. En este caso, los países desarrollados poseen el capital y una mayor riqueza de conocimientos técnicos; los países en desarrollo cuentan con un gran surtido de energía solar primaria, necesidades ilimitaclas de energia y costos menores de investigación.

Por último, en la medida que todos los paises reduzcan su dependencia en el petróleo como fuente de energía, tendrán más tiempo a su disposición y libertad cle acción para efectuar los dificilísimos cambios que serán indispensables para desplazarse hacia una economía energética no basada en el petróleo. Pero para que los beneficios de un tal enfoque sean recíprocos, los países industrializados -y especialmente los Estados Unidos- deben adoptar las medidas necestrias para contener los actuales sistemas dispencliosos e ineficientes de uso energético interno, a fin de fomentar una distribución más equitativa de los suministros petroleros disponibles.

Población.-La población mundial está creciendo a una tasa anual poco inferior al dos por ciento y la presente población de $4.000 \mathrm{mi}$ llones de habitantes se cree que alcanzará los 6.000 millones en el año 2000, para llegar a los 11.000 millones en el segundo decenio del siglo xxi, a no ser que se adopten medidlas para frenar el crecimiento de la población.

Tanta gente, casi tres veces cl número actual de habitantes de la tierra, forzará al máximo la capacidad de sustentación del planetri. Este crecimiento rápido de la población está causando ya y causarí ciertamente en el futuro, algunos problemas muy serios.

Hace más intensa la presión sobre la alimentación y los suministros energéticos mundiales. Obliga a utilizar cada vez más los recursos no renovables del plancta y es probable que aumente el número, y quizás incluso empeore la situación de los habitantes más pobres del mundo.

Organizar la coexistencia de algo así como $1 \cdot 1.000$ millones de personas, especialmente si aumentan los niveles de consumo, exigirá cambios cle gran alcance en las instituciones políticas, económicas y sociales existentes en el mundo. La estabilización de la población mundial al nivel más bajo posible interesa tanto a los países ricos como a los pobres.

La mayoria de los observadores concuertan en afirmar que la clave para limitar el crecimiento de la población estriba en satisfacer las necesidades socioeconómicas de la mayoría de la población pobre de los países en desarrollo. Es necesario aumentar la propaganda de las familias pequeñas, que es un requisito previo para la reducción de las tasas de natalidad.

Los programas que reducen las tasas de mortalidad infantil, ele- 
van los niveles de educación, aumentan las oportunidades y los ingresos de los grupos de baja renta y elevan la condición de la mujer, revisten importancia particular para conseguir tan necesaria motivación.

Las politicas que benefician el bienestar físico de la mayoría de la gente pobre de casi todos los países en desarrollo, son afortunaclamente las políticas destinadas al máximo incremento de la proclucción alimentaria en los países en clesarrollo, mediante la utilización intensiva de mano de obra y al aumento del suministro de energía rural de fuentes renovables.

Se puede acelerar mucho la reducción de las tasas mundiales de natalidad en un momento en que puecle alcanzarse la estabilización de la población en cada uno de los países. Esto se conseguirá combinando programas de planificación familiar en gran escala y bien claboraclos con programas que presten atención especial a mejorar el bienestar de la mayoría de la gente pobre de los paises en desarrollo.

Los países ricos $y$ pobres tienen el mismo interés en plantear en coopsración el problema de la población mundial. Muchos países en desarrollo tienen grancles dificultales para satisfacer las necesidacles humanas básicas de sus poblaciones actuales, y tendráll muchas más con el número mucho mayor de habitantes que se acumulará inevitablemente si no se plantea correctamente el probicmi.

Los países industriales tienen un interés directo en conseguir una población global munejable por razones de justicia y de igualdad muncliales $y^{\prime}$ porque las tensiones $y^{\prime}$ esfuerzos resultantes para el medio ambiente del planeta en sus vertientes políticas, económicas, sociales y físicats, plantearán probabiemente algunas dificultades casi insuperabies antes del linal de siglo, y ulteriormente.

El problema que está suscitando una creciente atención en los Estados Unidos es el de la politica de migraciones, principalmente de México y El Caribe, pero que afecta también il Europa Occidental y al Medio Oriente, en donde los paises productores de petróleo cstán importando trabajaclores del subcontinente asiático y hasta de Corea del Sur.

Los inmigrantes, seall lcgales o ilegales, aportan una diversiclad de beneficios cconómicos, ya que traen lécnicas y talentos, y' además cubren los puestos menos atractivos y peor pagados que no interesan a los trabiljadores localcs. Pero atcarrean también costos socioeconómicos, incluyendo las tensiones que surgen por la presencia de un gran número de extranjeros en otro pais.

Los Estados Unidos parecen dar prioridad a una doble política consistente en cerrar las fronteras y' en regularizar progresivamente la situación cle los extmanjeros, tanto legales como ilegales, que se encuentran ya en el país. 
Sin embargo, algunos analistas opinan que la única manera de enfrentar la inmigración ilegal, al menos en los Estados Uniclos, es atacar el problema de raiz. En otras palabras, que los movimientos migratorios de los paises pobres a los paises r:cos no disminuirán hasta que los nexicanos y otros emigrantes no tengan perspectivas de llevalr una vida decente en sus propios paises.

Wayne Comelius, clel Massachusetts Institute of Technology, estima que el método menos costoso a largo plazo para reducir el flujo de lá inmigración a los Estados Unidos consistiría en ampliar los programas existentes de desarrollo rural integrado que está Ilevando a cabo en México el Banco Mundial, el Banco Interamericano de Desatrollo y el gobiemo mexicano. Tales programas estín centraclos en el aumento de los empleos en México mecliante la promoción de industrias rurales en pequeña escala $y$ lia ulilización intensiva de mano de obra.

Una potenciación de los programas ubicalos en las zonas de México de donde procede la mayor parte de la emigración clandestina, combinado con un programit en grande escala de desarrollo rural por parte del gobierno mexicano, podria tener, según Cornelius, "una repercusión importante" en los esfucros por atajar la marea de inmigrantes, probablemente en un plazo que oscilaria entre los cinco $Y^{\prime}$ los diez : 1 ños" 20.

Una promesa por parte de los Estados Unidos de una mayor liberalización del comercio con México, permitiria una expansión rápicla del empleo en las industrias de exportación mexicanas y además el que muchos mexicanos llevaran una vida más decente y digna en su propio país.

Deberia considerarse la posibilidad de invertir en los programas de este tipo del gobierno mexicano, frente a las propuestats actuales de los je.uu. de cletener la oleada de inmigrantes con medidas restrictivas.

\section{¿TUEDE EL SUR CONVERTIRSE EN EL "MOTOR DE CRECIMIENTO" DEL NORTE?}

El análisis prececlente es aún excesivamente especulativo. Es necesnrio ahondar mucho mis en el trabajo analítico para determinar las ramificaciones completas de la dependencia de los pitises industriales respecto del crecimiento $y^{\prime}$ el progreso de los países en clesarrollo, y traducirlas en recomendaciones politicas concretas.

No obstante, las indicaciones son claras: cl crecimicnto futuro y el bienestar del mundo industrial dependerán de lo que ocurra en

3 WAYNe A. Conneisus, Undocumenled Inmigration: A Critique of the Carter Administration's Policy' Proposals, "Migration Today" Oct. 1977. 
el mundo en desarrollo; por lo tanto, trabajar en cooperación mutua brinda la única promesa real cle progreso para todos.

Si el análisis presentadlo en este documento es correcto, no es posible ya proseguir con las politicas actuales gae tienden a operar cambios marginales en las instituciones y politicas existentes, si se quiere que los sistemas politicos $y^{\prime}$ 'cconómicos mundiales funcionen eficaz y' equitativamente para los países rícos, por no hablar ya de los pobres.

Por consiguiente, hay que prestar scria atención a la idea de que cl desarrollo de los páses del Sur puecla convertirse en "el motor" tel crecimiento econónico mundial futuro, así como la recuperación plan:ficada de los países europeos y el establecimiento después de la Segunda Guema Mundial del sistema de instituciones financieras de Bretton Woods se convirtió en el "motor" del progreso económico giobal sin precedentes que se llevó a cabo en los años 50 y 60 . Poniendo el progreso de los países en clesarrollo como centro de sus politicas económicas, los paises industrializados podrían asegurar igualmente el progreso futuro del Norte y el Sur.

Es más, según los datos presentaclos en cste clocumento, parece probable que la elección entre continuar las políticas presentes $y^{\prime}$ proceder a un esfuerzo de mayor alcance para ajustar las politicas $y$ las instituciones a las necesidades del mundo en los próximos 25 años, podria significar una diferencia de tasas de crecimiento eco. nómico en algunos de Jos países desarrollados de un 1 ó un 2 por ciento al año. En el caso de los patises desarrollaclos, la diferencia del 2 al 4 por ciento de la tasa de crecimiento en los años restantes cle esta centuria, supondria un ingreso per cápita en el año 2000 de unos 5.600 clólares más $y$ un incremento clel I'Ns que seria equivalente al PNB de los paises de la OCDE en 1975.

Un esfuerzo semejante podria representar un modelo de desarrollo para los Estados Unidos y otros países industriaies que claramente siguen un mismo proceso, caracterizado por la baja inflación y el elevado desempleo que se repiten constantemente a mitad cle los años 70 .

Lo que se necesita es una serie cle politicas globules que tengan como punto central el progreso de los países en clesarrollo, tanto porque es una meta importante en sí misma, como por su repercusión en el progreso futuro de los paises en desarrollo.

Este criterio: 1) claria prioridad a reformas amplias de las estructuras y políticas internacionales existentes, para acrecentar la eficiencia y la equidad del orden económico internacional, y 2) intentaría satisfacer las necesidades esenciales de los paises y pueblos más pobres del mundo para finales de este siglo 21 .

${ }^{21}$ Se presentaron recomendaciones y anilisis detallados en The United States 
Este cmpeño exige cambios de las politicas actuales, que habrán de ser objeto de negociación, a fin de dar a los países en desarrollo mayor acceso a los mercados de los países industriales; estabilizar los precios de las materias primas y los productos básicos; crear una serie de nuevos sistemas globales para regular la proclucción y la clistribución de alimentos y energía; y mejorar el acceso de los países en desarrollo a una variedad de fuentes de financiación nuevas $y$ existentes.

Esta acción englobará a ricos y pobres en una operación de cooperación para satisficer las necesidades humanas básicas de los millones y millones de pobres del mundo hacia finales de este siglo.

Se tratará de alcanzar los modelos internacionalmente acordados de nutrición, servicios sanitarios y educación básica y para su éxito será menester que se comprometan los propios países en desarrollo. Los países productores de petróleo deberán desempeñar un importante papel de cooperación en apoyo de esas políticas.

El "paquete" de reformas alterará el sistema internacional y resolverá el problema de las necesidades humanas, servirá a los intereses cle los países en desarrollo de ingresos medios (que recibirán muchos de los beneficios de las reformas internacionales), así como a los de los paises en desarrollo de ingresos bajos (que se enfrentan aún con problemas de pobreza masiva y que necesitarán transferencias en condiciones de favor para satisfacer las necesidades básicas de grancles sectores de sus poblaciones) y atenderá también a las necesidades de los paises inclustriales en pro de una economía internacional más eficiente y en crccimiento, que contribuya a su propia expansión por medio del estímulo de la oferta y de la demandia en el mundo en clesarrollo.

Por último, es importante acoplar los clos aspectos de la reforma, ya que satisface la exigencia primordial por parte de los países en clesarrollo de unas relaciones políticas y económicas más equitativas entre los estados y calma la preocupación de muchos paises industrializacios por la repercusión de los cambios en las poblaciones de Jos países desarrollados y en clesarrollo.

Sin embargo, no cleben subestimarse los costos posibles a corto plazo en los paises desarrollatios $y$ entre los mismos, incluyendo a los Estados Unidos, que podrín surgir de este conjunto de politicas.

Aumentar el acceso de los paises en desarrollo a los mercados de los paises desarrollaclos afectaríi a los empleos y a las empresas en ciertas inclus trias.

Los costos económicos del reajuste equitativo de los programas de

and World Development, Agenda 1977. Sewell $y$ el Personal del Consejo del Oversens Development Council, Pracger, N. York. 
asistencia no es probable que sean grandes, pero los costos políticos pueden a veces ser altos a causa cle la oposición cle grupos organi7ados eficazmente. También la financiación que necesitan los países en desarrollo para sufragar sus deudas y mantener el ritmo del. desarrollo entraña costos presupuestarios.

Podrán existir también algunos rićsgos, cambios de progranas y quizás incumplimientos si los prestamistas contintaun financiando a los paises en desarrollo.

Entre los paises de la OCDE los costos pueden variar. Los Estaclos Unidos y Catnadá, que son almacenes continentales cle materiạs primas y que tienen grandes mercados internos, no están en la misma posición que Japón y los países europeos que dependen del mundo en clesarrollo, tanto para los mercados como para el súministro de una variedad de materias. Por último, estas politicas exigirán visión y capacidad dirigente, cualidades que han brillado por su ausencia en el mundo industrial, no sólo por parte de los políticos sino del público en general.

Pero los costos de no tratar de establecer un orden mundial mutuamente beneficioso serán altos, y, en cambio, los beneficios serian considerables. Se precisa una labor de cooperación que conceda prioridad a una mayor eficacin de los sistemas económicos mundiales, asi como una mayor equidad $y$ justicia.

No hay duda de que la reserva de productividad marginal mayor del mundo está constituida por los recursos físicos y humanos, aún inexplotados e infrautilizados, de los paises en tlesarrollo.

Aumentando el poder adquisitivo de los habitantes de aquellos países, liberando nuevos recursos $y$ prospectindo nuevos mercados tanto para ricos como para pobres, puede iograrse un nivel mayor cle comercio entre los paises y en el interior cle los mismos. En efec: to, los paises en desarrollo del Sur podrían convertirse en uno de los motores de la reactivación del crecimiento y el progreso en el mundo industrial.

Por consiguiente, volvemos a nucstra aserción inicial. El logro de las metás económicas nacionales de los países desarrollados -reemprender el crecimiento, aumentar los empleos y. lograr precios más estables- dependerá más que nunca : del crecimiento y la prospericlad de los paises en desarrollo:

Los formuladores cle políticas y el público informado de éste pais, de otros paises industrializados y de los países en desarrollo, han de reconocer $y$ tomar en consicleración este factor. 